\title{
Modeling of microdischarge devices: Pyramidal structures
}

\author{
Mark J. Kushnera) \\ Department of Electrical and Computer Engineering, University of Illinois, 1406 W. Green Street, \\ Urbana, Illinois 61801
}

(Received 23 June 2003; accepted 30 October 2003)

\begin{abstract}
Microdischarge (MD) devices are plasma sources typically operating at 100s Torr to atmospheric pressure with dimensions of $10 \mathrm{~s}-100 \mathrm{~s} \mu \mathrm{m}$. Their design in based on $p d$ (pressure $\times$ characteristic dimension) scaling; smaller dimensions are enabled by higher operating pressures with typical devices operating with $p d=1-10$ Torr $\mathrm{cm}$. MD devices have exhibited behavior that resemble both Townsend and hollow-cathode discharges, with bulk and beam electrons providing the dominant excitation, respectively. In this article, results from a two-dimensional computational study of MD devices operating in neon using a pyramidal cathode structure are discussed. Pressures of 400-1000 Torr and device dimensions of 15-40 $\mu \mathrm{m}$ are investigated. The onset of behavior resembling negative glow discharges with decreasing pressure correlates with an extension of cathode fall accelerated beam electrons into the bulk plasma. For constant applied voltage, peak electron densities increase with increasing pressure as the beam electrons are slowed in more confined regions. The MD devices typically require higher applied voltages to operate at lower pressures, and so resemble discharges obeying Paschen's curve for breakdown. MD devices having similar magnitudes and spatial distributions of plasma and excited state densities can be obtained to dimensions of $<15 \mu \mathrm{m}$ by keeping $p d$ and current density constant, and having a cathode fall thickness small compared to the characteristic dimension. () 2004 American Institute of Physics. [DOI: $10.1063 / 1.1636251]$
\end{abstract}

\section{INTRODUCTION}

Microdischarge (MD) devices take advantage of the $p d$ (pressure $\times$ electrode separation) scaling of low temperature partially ionized plasmas to operate stably as glow discharges at pressures approaching and exceeding atmospheric with dimensions as small as $10 \mathrm{~s} \mu \mathrm{m} .{ }^{1-9}$ MDs having characteristic dimensions of tens to hundreds of $\mu \mathrm{m}$ have been developed which operate at 100s Torr to as much as 1000 Torr in rare gas, rare gas-halogen, and molecular gas mixtures on a continuous basis. Early variants of MDs were constructed with the intent to operate as miniature hollow cathodes by reducing the cathode diameter while increasing the pressure to maintain the same $p d$ as conventional low pressure hollow cathode devices. ${ }^{10}$ Observations of optical emission spectra showed that when $p d$ decreased into the 1-10 Torr $\mathrm{cm}$ range, the devices transitioned from operating similar to a Townsend discharge dominated by emission from neutral atoms to a mode resembling a hollow cathode discharge in which emission from high lying ion transitions was prominent. ${ }^{1}$ Employing techniques developed for microelectronics and microelectromechanical systems fabrication, MD structures have been investigated. Of particular interest are inverted pyramidal structures, and arrays of these structures, fabricated in silicon substrates. ${ }^{1}$

MD devices have been studied extensively in the context of plasma display panels (PDPs). (See, for example, Ref. 11 for a recent review.) MD devices for PDPs differ from those of interest here in a number of regards. PDP plasma cells are

${ }^{a)}$ Electronic mail: mjk@uiuc.edu intrinsically pulsed devices due to their use of dielectrically coated electrodes. PDP cells also tend to have somewhat larger dimensions $(100 \mathrm{~s} \mu \mathrm{m})$ compared to the $10 \mathrm{~s} \mu \mathrm{m}$ of interest here. Whereas $\mathrm{cw}$ MD devices tend to rely upon beam components of the electron energy distribution for ionization, PDP devices are likely more reliant upon Townsend processes. Although there are strong similarities between the physics, technology and operation of PDP and cw MD devices, we will restrict discussion here specifically to continuous operating MD devices which are not of the conventional PDP design.

Although MD devices are often described as microhollow-cathodes, they lack many of the features of conventional hollow cathodes. Conventional hollow cathodes typically have opposing parallel cathode surfaces which enable secondary electrons accelerated through the cathode fall to undergo pendular motion between the cathode sheaths. The compression of the slowing down length of beam electrons into the smaller dimensions of the negative glow between the cathodes increases the power density compared to conventional negative glows and produces the high plasma densities associated with hollow cathodes. In devices without clearly opposing cathodes, similar transitions between behavior resembling Townsend discharges and hollow cathode discharges can occur, but likely without the increase in power density typically associated with the hollow-cathode effect. In devices truly taking advantage of the hollow cathode effect, beam electrons accelerated in the cathode fall and reciprocating between the geometrically opposing cathode falls deposit their energy in a smaller volume and hence increase the power density. 
Classical scaling of normal glows states that cathode fall voltage will remain constant provided that $p d$ remains constant. Under these conditions, current density scales as $p^{2}$. In principle, $p d$ scaling should enable MD devices to be scaled to dimensions of only a few microns, the mean free path of electrons at near atmospheric pressure. (Note that in multidimensional devices, $p d$ scaling implies that all dimensions should scale inversely with pressure. In this regard, we will use $d$ as representative of the scale length of the MD device.) This scaling assumes that the sheath or cathode fall thickness, $\lambda$, is small compared to $d$. $P d$ scaling is questionable when $d$ decreases to be commensurate with $\lambda$ and a fully developed cathode fall cannot be accommodated within the MD structure. At this juncture, power densities must be increased sufficiently to increase the electron density and reduce $\lambda$ to fit the confines of the device.

In this article, results from a computational investigation of the scaling of MD devices are discussed. The MDs we studied use an inverted pyramidal cathode with diameters of 25-100 $\mu \mathrm{m}$ patterned after those experimentally investigated by Park et al. ${ }^{1,4}$ We found that these devices, when operated in neon, are largely sustained by ionization by beam electrons accelerated in the cathode fall. Regions of sufficiently high plasma density are produced to confine the cathode fall to the dimensions of the cathode cavity. MDs will not operate on a cw basis for conditions which cannot produce this critically large plasma density. The highest electron densities observed, however, are insufficient to produce fully shielded plasmas within the entire volume of the device. The end result is that truly quasi-neutral plasma volumes are generally not produced throughout the device. The model will be described in Sec. II followed by a discussion of the results from our study in Sec. III. Concluding remarks are in Sec. IV.

\section{DESCRIPTION OF THE MODEL}

The model employed in this study is based on LAMPSIM, which is described in detail in Ref. 12. LAMPSIM will be briefly described here with emphasis on improvements. LAMPSIM, is a multifluid, two-dimensional hydrodynamics simulation in which transport equations for all charged and neutral species, and Poisson's equation, are integrated as a function of time. The numerical grid uses a boundary fitting, unstructured mesh with triangular elements. The method of solution uses time-slicing techniques between charged particle transport and neutral particle transport updates. The time evolution of charged particle densities and surface charges are solved simultaneously with Poisson's equation using an implicit Newton's method. This update is then followed with an implicit update of neutral particle densities and electron temperature; and less frequent transport of beam electrons and update of the gas temperature.

The numerical mesh for LAMPSIM was produced using SkyMesh $2,{ }^{13}$ a commercially available mesh generator. The mesh consisted of triangles, using mesh relaxation techniques to optimize angles close to $60^{\circ}$ and eliminate obtuse angles whereever possible. The resolution of the mesh was selected to resolve both geometrical structures and regions of large electric field gradients or large particle density gradients with a fine mesh, while using a coarse mesh in outlying regions. The differential equations discussed below were discretized on the mesh using finite volume techniques.

The fundamental algorithms employed for plasma transport in LAMPSIM are

$$
\begin{aligned}
& -\nabla \cdot(\varepsilon \nabla \Phi)=\sum_{j=1}^{n} q_{j} N_{j}+\rho, \\
& \frac{\partial N_{i}}{\partial t}=-\nabla \cdot \phi_{i}+S_{i}, \\
& \frac{\partial \rho}{\partial t}=\left[\sum_{j=1}^{n} q_{j}\left[-\nabla \cdot \phi_{j}+S_{j}\right]-\nabla \cdot(\sigma(-\nabla \Phi))\right]_{m},
\end{aligned}
$$

where $\varepsilon, \Phi, q, N, \rho, S$, and $\sigma$ are permittivity, electric potential, elementary charge, species number density, charge density, source function due to collisions, and conductivity of solid materials, respectively. The subscript denotes the identity of the species. $\phi$ is the species flux accounting for both drift in the electric field and diffusion due to density gradients. $n$ is the total number of charged gas species (electrons and ions). These expressions are Poisson's equation [Eq. (1)], continuity for charged species [Eq. (2)] and continuity for charges on surfaces and in materials [Eq. (3)]. The source function in Eq. (2) includes the gain and loss terms due to gas phase reactions (neutral chemistry, ion-molecule reactions, and electron impact processes) and contributions associated with wall chemistry, including electron emission from surfaces. The brackets and subscript $m$ in Eq. (3) denote that $\rho$ is only computed on surfaces and in bulk materials.

The flux terms for charged species $\phi$ were formulated using the method of Scharfetter and Gummel. ${ }^{14}$ In this method, the flux $\boldsymbol{\varphi}_{i+(1 / 2)}$ between density mesh points $(i, i$ +1 ) separated by a distance $\Delta x$ is given by

$$
\boldsymbol{\varphi}_{i+1 / 2}=\frac{\alpha \bar{D}\left[n_{i+1}-n_{i} \exp (\alpha \Delta x)\right]}{[1-\exp (\alpha \Delta x)]},
$$

where

$$
\alpha=-q \bar{\mu}\left(\frac{\Phi_{i+1}-\Phi_{i}}{\Delta x}\right)
$$

and $\bar{D}$ and $\bar{\mu}$ are the average diffusion coefficient and mobility in the interval. This method has the property of being upwind (or downwind) in accordance with the direction and magnitude of the drift flux compared to the diffusion flux.

All Laplacian operators are formulated using conservative finite volume techniques. For example, for node $i$ and flux $\phi$

$$
-\nabla \cdot \phi_{i}=-\frac{1}{V_{i}} \sum_{k=1}^{n} \frac{A_{i, k} \phi_{i, k}}{\left|\mathbf{r}_{i}-\mathbf{r}_{k}\right|},
$$

where $\phi_{i, k}$ is the flux between nodes $i$ and $k$ defined as being positive if directed away from $i, \mathbf{r}_{i}$ is the spatial location of node $i, A_{i, k}$ is the area of the face between the volume cells centered on nodes $i$ and $k$, and $V_{i}$ is the volume of the cell for node $i$. The system of equations for charged particle 
transport and Poisson's equation is integrated in time using an implicit Newton's method with numerically derived Jacobian elements. The resulting sparse matrix was solved using the numerical package dslucs, obtained from the SLAP Sparse Matrix Library. ${ }^{15,16}$ The matrix solver uses a biconjugate gradient spare matrix solution technique with incomplete LU factorization for preconditioning.

In a time-splicing manner, following updates of the charged particle densities, the neutral particle densities are next implicitly updated using the expression

$$
\begin{aligned}
N_{i}(t+\Delta t)= & N_{i}(t)-\nabla \cdot\left(\boldsymbol{\nu}-N_{0} D_{i} \nabla\left(\frac{N_{i}(t+\Delta t)}{N_{0}}\right)\right) \Delta t \\
& +S_{i} \Delta t,
\end{aligned}
$$

where $\boldsymbol{\nu}$ is the fluid averaged advective velocity, $D_{i}$ is the diffusion coefficient and $N_{0}$ is the total gas density. Equation (7) is sequentially solved for each species using the method of successive over-relaxation (SOR) with the Laplacian terms formulated using finite volume techniques.

Electron impact rate and transport coefficients for bulk electrons were obtained by solving the electron energy conservation equation for average electron energy $\varepsilon$

$\frac{\partial\left(n_{e} \varepsilon\right)}{\partial t}=\mathbf{j} \cdot \mathbf{E}-n_{e} \sum_{i} N_{i} \kappa_{i}-\nabla \cdot\left(\frac{5}{2} \varepsilon \phi_{e}-\lambda_{e} \nabla T_{e}\right), \quad \mathbf{j}=q \boldsymbol{\phi}_{e}$

where $T_{e}$ is the electron temperature [defined as $(2 / 3) \varepsilon$ ], $n_{e}$ is the electron density, $\kappa_{i}$ is the rate coefficient for power loss $\left(\mathrm{eV} \mathrm{cm}^{3} \mathrm{~s}^{-1}\right)$ for collisions of electrons with species $i$ having density $N_{i}, \lambda_{e}$ is the electron thermal conductivity, and $\phi_{e}$ is the electron flux [obtained from Eq. (4)]. This equation is also implicitly solved as a time integration using a SOR technique. An electron temperature of $0.05 \mathrm{eV}$ is assigned to all surfaces in contact with the plasma and the thermal conductivity is assigned appropriate values across the sheath commensurate with the electron density in the sheath. This effectively results in an adiabatic boundary condition. The electron transport coefficients and rate coefficients for use in solving Eq. (8) are obtained by solving Boltzmann's equation for the electron energy distribution using a two-term spherical harmonic expansion, as when using the local field approximation. Boltzmann's equation is parameterized over a range of $E / N$, and a table of transport coefficients as a function of $\varepsilon$ is constructed. This table is then interpolated during solution of Eq. (8).

Surface chemistry is included using a flux-in/flux-out boundary condition. For each species, a "disappearance" coefficient $\alpha_{i}$ and production coefficients $\beta_{i, k}$ are specified where $\alpha_{i}$ is the probability that species $i$ is consumed by the surface and $\beta_{i, k}$ is the rate of production of species $k$ by species $i$. The returning flux from the surface is then

$$
\phi_{i}=\phi_{i}^{o}\left(1-\alpha_{i}\right)+\sum_{k} \phi_{k}^{o} \beta_{k, i},
$$

where $\phi_{i}^{o}$ is the flux into the surface obtained assuming the surface density is zero and the sum is over other species. The coefficient for secondary electron emission used here was 0.15 for all ions.
As specific power deposition in microdischarges can be many $100 \mathrm{~s} \mathrm{~kW} / \mathrm{cm}^{3}$ gas heating and rarefaction can be expected to be important. To assess these effects a simple heat conduction model was employed to obtain the gas temperature $T_{g}$,

$$
\frac{\partial\left(\rho c_{p} T_{g}\right)}{\partial t}=P_{g}+\nabla \cdot \lambda_{g} \nabla T_{g}
$$

where $P_{g}$ is the power deposited into the translational modes of the gas, $\lambda_{g}$ is the mole fraction averaged thermal conductivity, and $c_{p}$ is the specific heat. $P_{g}$ has contributions from ion Joule heating $(\mathbf{j} \cdot \mathbf{E})$, increments to enthalpy due to heavy particle reactions (e.g., Frank-Condom heating and chemical reactions) and by elastic electron collisions, though the latter contribution is small. The temperature on the outer boundary of the mesh was held constant at $300 \mathrm{~K}$. As convective heat transfer is being ignored, this approach provides the maximum dynamic range in gas temperature and so can be considered a worst-case-analysis for the consequences of gas heating on device performance. As we are only concerned with steady state operating characteristics, Eq. (10) was implicitly integrated using time steps many times larger than those used for update of the plasma properties. The advective velocity in Eq. (7) was set to zero and the total gas densities renormalized assuming isobaric ideal gas behavior.

A simple circuit model is employed to provide driving voltages where all metal surfaces are connected to ground through a ballast resistor and, optionally, a power supply. The electric potential boundary conditions for solution of Poisson's equation are obtained from the circuit model and are applied to biased metal surfaces. Currents through each leg of the circuit are obtained by summing charged particle fluxes. Electrically floating metal surfaces are actually represented by dielectrics having sufficiently high conductivities and permittivities that they appear to be equipotential surfaces.

The transport of secondary beam electrons emitted from the cathode are tracked using an electron Monte Carlo Simulation (eMCS). The fundamentals of the eMCS will briefly be described. The computational mesh employed in the plasma hydrodynamic portion of the model is unstructured. As such, it is computationally expensive to locate particles in the mesh during the eMCS to accumulate statistics at nodes in the mesh; or to obtain mesh quantities, such as electric fields and collision frequencies, required to advance the trajectories of the pseudoparticles. As these assignments and interpolations would otherwise be responsible for the vast majority of the computer time spent in the eMCS, the following methodology was used.

The advancement of trajectories in the eMCS is performed on a Cartesian mesh (CM) which is overlayed onto the unstructured hydrodynamics mesh (UM). The CM overlays only that portion of the UM in which beam electron transport is expected to be important, a choice refined by iteration and experience. The resolution of the $\mathrm{CM}$ is chosen to be fine enough to capture the small scale features of the UM. As the number of arrays in the eMCS which are indexed on the CM is small, there is not a large computational 
TABLE I. Species in the model and reaction mechanism.

\begin{tabular}{|c|c|c|}
\hline $\begin{array}{l}\text { Reaction mechanism } \\
\text { Reaction }\end{array}$ & Rate coefficient ${ }^{\mathrm{a}}$ & Reference \\
\hline \multicolumn{3}{|l|}{ Electron impact processes } \\
\hline$e+\mathrm{Ne} \rightarrow \mathrm{Ne}+e$ & $\mathrm{~b}$ & 18 \\
\hline$e+\mathrm{Ne} \rightarrow \mathrm{Ne}\left(1 s_{35}\right)+e$ & $\mathrm{~b}$ & 19 \\
\hline$e+\mathrm{Ne} \rightarrow \mathrm{Ne}\left(1 s_{24}\right)+e$ & $\mathrm{~b}$ & 19 \\
\hline$e+\mathrm{Ne} \rightarrow \mathrm{Ne}(2 p)+e$ & $\mathrm{~b}$ & 20 \\
\hline$e+\mathrm{Ne} \rightarrow \mathrm{Ne}^{+}+e+e$ & $\mathrm{~b}$ & 21 \\
\hline$e+\mathrm{Ne} \rightarrow \mathrm{Ne}^{++}+e+e+e$ & b & 21 \\
\hline$e+\mathrm{Ne}\left(1 s_{35}\right) \rightarrow \mathrm{Ne}\left(1 s_{35}\right)+e$ & $\mathrm{~b}$ & c \\
\hline$e+\mathrm{Ne}\left(1 s_{35}\right) \rightarrow \mathrm{Ne}(2 p)+e$ & $\mathrm{~b}$ & 22 \\
\hline$e+\mathrm{Ne}\left(1 s_{35}\right) \rightarrow \mathrm{Ne}+e$ & $\mathrm{~b}$ & d \\
\hline$e+\mathrm{Ne}\left(1 s_{35}\right) \rightarrow \mathrm{Ne}^{+}+e+e$ & $\mathrm{~b}$ & 23 \\
\hline$e+\mathrm{Ne}\left(1 s_{24}\right) \rightarrow \mathrm{Ne}\left(1 s_{24}\right)+e$ & $\mathrm{~b}$ & $\mathrm{c}$ \\
\hline$e+\mathrm{Ne}\left(1 s_{24}\right) \rightarrow \mathrm{Ne}(2 p)+e$ & b & 22 \\
\hline$e+\mathrm{Ne}\left(1 s_{24}\right) \rightarrow \mathrm{Ne}+e$ & $\mathrm{~b}$ & d \\
\hline$e+\mathrm{Ne}\left(1 s_{24}\right) \rightarrow \mathrm{Ne}^{+}+e+e$ & $\mathrm{~b}$ & 23 \\
\hline$e+\mathrm{Ne}(2 p) \rightarrow \mathrm{Ne}(2 p)+e$ & b & $\mathrm{c}$ \\
\hline$e+\mathrm{Ne}(2 p) \rightarrow \mathrm{Ne}+e$ & $\mathrm{~b}$ & d \\
\hline$e+\mathrm{Ne}(2 p) \rightarrow \mathrm{Ne}\left(1 s_{35}\right)+e$ & $\mathrm{~b}$ & d \\
\hline$e+\mathrm{Ne}(2 p) \rightarrow \mathrm{Ne}\left(1 s_{24}\right)+e$ & $\mathrm{~b}$ & d \\
\hline$e+\mathrm{Ne}(2 p) \rightarrow \mathrm{Ne}^{+}+e+e$ & b & 24 \\
\hline$e+\mathrm{Ne}\left(1 s_{24}\right) \rightarrow \mathrm{Ne}\left(1 s_{35}\right)+e$ & $7 \times 10^{-8} T_{e}^{0.5}$ & $\mathrm{e}$ \\
\hline$e+\mathrm{Ne}\left(1 s_{35}\right) \rightarrow \mathrm{Ne}\left(1 s_{24}\right)+e$ & $7 \times 10^{-8} T_{e}^{0.5} \exp \left(-0.06 / T_{e}\right)$ & $\mathrm{e}$ \\
\hline$e+\mathrm{Ne}_{2}^{*} \rightarrow \mathrm{Ne}_{2}^{+}+e+e$ & $9.75 \times 10^{-9} T_{e}^{0.71} \exp \left(-3.4 / T_{e}\right)$ & 25 \\
\hline$e+\mathrm{Ne}_{2}^{*} \rightarrow \mathrm{Ne}+\mathrm{Ne}+e$ & $1.0 \times 10^{-7}$ & 26 \\
\hline$e+\mathrm{Ne}_{2}^{+} \rightarrow \mathrm{Ne}(2 p)+\mathrm{Ne}$ & $3.7 \times 10^{-8} T_{e}^{-0.5}$ & 27 \\
\hline$e+\mathrm{Ne}^{+} \rightarrow \mathrm{Ne}(2 p)$ & $4.0 \times 10^{-13} T_{e}^{-0.5}$ & 28 \\
\hline$e+e+\mathrm{Ne}^{+} \rightarrow \mathrm{Ne}(2 p)+e$ & $5.0 \times 10^{-27} T_{e}^{-4.5}$ & 28 \\
\hline \multicolumn{3}{|l|}{ Heavy particle reactions } \\
\hline $\mathrm{Ne}\left(1 s_{24}\right)+\mathrm{Ne}\left(1 s_{24}\right) \rightarrow \mathrm{Ne}^{+}+\mathrm{Ne}+e$ & $3.2 \times 10^{-10}$ & $29^{\mathrm{f}}$ \\
\hline $\mathrm{Ne}\left(1 s_{24}\right)+\mathrm{Ne}\left(1 s_{35}\right) \rightarrow \mathrm{Ne}^{+}+\mathrm{Ne}+e$ & $3.2 \times 10^{-10}$ & $29^{\mathrm{f}}$ \\
\hline $\mathrm{Ne}\left(1 s_{24}\right)+\mathrm{Ne}(2 p) \rightarrow \mathrm{Ne}^{+}+\mathrm{Ne}+e$ & $3.2 \times 10^{-10}$ & $29^{\mathrm{f}}$ \\
\hline $\mathrm{Ne}\left(1 s_{35}\right)+\mathrm{Ne}\left(1 s_{35}\right) \rightarrow \mathrm{Ne}^{+}+\mathrm{Ne}+e$ & $3.2 \times 10^{-10}$ & $29^{\mathrm{f}}$ \\
\hline $\mathrm{Ne}\left(1 s_{35}\right)+\mathrm{Ne}(2 p) \rightarrow \mathrm{Ne}^{+}+\mathrm{Ne}+e$ & $3.2 \times 10^{-10}$ & $29^{\mathrm{f}}$ \\
\hline $\mathrm{Ne}(2 p)+\mathrm{Ne}(2 p) \rightarrow \mathrm{Ne}^{+}+\mathrm{Ne}+e$ & $3.2 \times 10^{-10}$ & $29^{\mathrm{f}}$ \\
\hline $\mathrm{Ne}_{2}^{*}+\mathrm{Ne}_{2}^{*} \rightarrow \mathrm{Ne}_{2}^{+}+\mathrm{Ne}+\mathrm{Ne}+e$ & $1.0 \times 10^{-11}$ & e \\
\hline $\mathrm{Ne}^{+}+\mathrm{Ne} \rightarrow \mathrm{Ne}+\mathrm{Ne}^{+}$ & $3.0 \times 10^{-10}$ & 30 \\
\hline $\mathrm{Ne}\left(1 s_{24}\right)+\mathrm{Ne}+\mathrm{Ne} \rightarrow \mathrm{Ne}_{2}^{*}+\mathrm{Ne}$ & $4.1 \times 10^{-34} \mathrm{~cm}^{6} \mathrm{~s}^{-1}$ & 26 \\
\hline $\mathrm{Ne}\left(1 s_{35}\right)+\mathrm{Ne}+\mathrm{Ne} \rightarrow \mathrm{Ne}_{2}^{*}+\mathrm{Ne}$ & $4.1 \times 10^{-34} \mathrm{~cm}^{6} \mathrm{~s}^{-1}$ & 26 \\
\hline $\mathrm{Ne}(2 p)+\mathrm{Ne}+\mathrm{Ne} \rightarrow \mathrm{Ne}_{2}^{*}+\mathrm{Ne}$ & $4.1 \times 10^{-34} \mathrm{~cm}^{6} \mathrm{~s}^{-1}$ & $26^{\mathrm{g}}$ \\
\hline $\mathrm{Ne}^{+}+\mathrm{Ne}+\mathrm{Ne} \rightarrow \mathrm{Ne}_{2}^{+}+\mathrm{Ne}$ & $4.4 \times 10^{-32} \mathrm{~cm}^{6} \mathrm{~s}^{-1}$ & 26 \\
\hline $\mathrm{Ne}\left(1 s_{24}\right)+\mathrm{Ne} \rightarrow \mathrm{Ne}\left(1 s_{35}\right)+\mathrm{Ne}$ & $4.2 \times 10^{-14}$ & 31 \\
\hline $\mathrm{Ne}\left(1 s_{35}\right)+\mathrm{Ne} \rightarrow \mathrm{Ne}\left(1 s_{24}\right)+\mathrm{Ne}$ & $3.4 \times 10^{-14}$ & 31 \\
\hline \multicolumn{3}{|l|}{ Radiative transitions } \\
\hline $\mathrm{Ne}_{2}^{*} \rightarrow \mathrm{Ne}+\mathrm{Ne}$ & $3.6 \times 10^{8} \mathrm{~s}^{-1}$ & 26 \\
\hline $\mathrm{Ne}(2 p) \rightarrow \mathrm{Ne}\left(1 s_{35}\right)$ & $1.84 \times 10^{7} \mathrm{~s}^{-1}$ & 31 \\
\hline $\mathrm{Ne}(2 p) \rightarrow \mathrm{Ne}\left(1 s_{24}\right)$ & $1.25 \times 10^{7} \mathrm{~s}^{-1}$ & 31 \\
\hline $\mathrm{Ne}\left(1 s_{24}\right) \rightarrow \mathrm{Ne}$ & $1.0 \times 10^{1} \mathrm{~s}^{-1}$ & $19,32^{\mathrm{h}}$ \\
\hline
\end{tabular}

${ }^{a}$ Rate coefficients have units of $\mathrm{cm}^{3} \mathrm{~s}^{-1}$ unless noted otherwise. $T_{e}$ is the electron temperature $(\mathrm{eV})$.

${ }^{b}$ Process was included for both bulk and beam electrons. Rate coefficients were determined by convolving the cross section from the indicated reference with the electron energy distribution obtained from solution of Boltzmann's equation (bulk electrons) or the eMCS (beam electrons).

${ }^{c}$ Elastic cross sections for excited states were estimated to be constant at $1.0 \times 10^{-14} \mathrm{~cm}^{2}$.

${ }^{\mathrm{d}}$ Cross section obtained by detailed balance.

${ }^{\mathrm{e}}$ Estimated.

${ }^{\mathrm{f}}$ Rate coefficient is for mutual Penning ionization of $\mathrm{Ne}(1 s)$. That value was used for Penning ionization between all excited states.

${ }^{\mathrm{g}}$ Estimated to have the same rate coefficient as for $\mathrm{Ne}(1 s)$.

${ }^{\mathrm{h}}$ Radiation trapped values are based on untrapped lifetimes of $1.65 \mathrm{~ns}$ for $\mathrm{Ne}\left(1 s_{2}\right)$ and $20.5 \mathrm{~ns}$ for $\mathrm{Ne}\left(1 s_{4}\right)$.

penalty to having a fine enough resolution in the $\mathrm{CM}$ to capture the resolution of UM.

At the beginning of the eMCS, Green's functions are developed for interpolation of quantities from the UM to the
$\mathrm{CM}$, and vice versa. To interpolate from the UM to a node in the CM, the nearest nodes from the UM in each of the four Cartesian quadrants centered on the node in the $\mathrm{CM}$ are located. Quantities on the UM in adjacent quadrants are inter- 
polated to the axes of the $\mathrm{CM}$; and those axial quantities are then interpolated to the central CM node. To interpolate from the $\mathrm{CM}$ to a node in the $\mathrm{UM}$, the mesh cell in the $\mathrm{CM}$ containing node in the UM is located. The four vertices of the cell in the CM then provide values to perform a twodimensional interpolation to the node in the UM. As such, the ideal spacing of the $\mathrm{CM}$ is to have a single node from the $\mathrm{UM}$ in each cell of the CM. In practice, this is not possible and so search algorithms are employed to locate the appropriate vertices in the $\mathrm{CM}$ and nodes in the UM to facilitate use of the Green's functions.

Secondary electron emission from surfaces is addressed using the eMCS. The electric potentials produced in the fluid module are interpolated to the $\mathrm{CM}$. Based on incident ion fluxes and secondary electron emission coefficients, electron pseudoparticles are released with an energy of $4 \mathrm{eV}$ from nodes on surfaces in the UM. These pseudoparticles are weighted by the magnitude of the local ion flux, the secondary electron emission coefficient, and the number of particles released at each node. The weighting of each pseudoparticle has units of electrons/s. Using the Monte Carlo techniques described in Ref. 17, the trajectories of the secondary beam electrons and their progeny are integrated as a function of time. Pseudoparticles (and their progeny) are tracked until they hit boundaries, move out of the confines of the $\mathrm{CM}$ or fall below a specified energy thereby joining the bulk electron distribution. The weightings of these latter pseudoparticles are summed into sources $S_{i}$ for electrons and are included in Eq. (1). The trajectories of the beam electrons are sampled with each move of the pseudoparticles, binning them in energy and location on the CM to produce spatially dependent electron energy distributions, $f(\varepsilon, \mathbf{r})$ having units of electrons $\mathrm{cm}^{-3} \mathrm{eV}^{-1}$. When convolved with electron impact cross sections, source functions having units $\mathrm{cm}^{-3} \mathrm{~s}^{-1}$ are produced which then contribute to $S_{i}$ for the appropriate species. These source functions are then interpolated onto the UM.

The time step between executing the eMCS varies from as short as $0.1 \mathrm{~ns}$ during the startup transient to $10 \mathrm{~s}$ ns during the steady state. The time steps between executions of the eMCS are specified as input to LAMPSIM, and are choices which are largely based on experience. As the plasma conditions approach the steady state, sources from the eMCS are often back averaged from iteration to iteration to reduce the consequences of numerical noise.

The investigations reported here used a pure Ne gas mixture using the reaction mechanism shown in Table I. The atomic model consisted of (Paschen notation) $\mathrm{Ne}$ (ground state), $\mathrm{Ne}\left(1 s_{24}\right), \mathrm{Ne}\left(1 s_{35}\right), \mathrm{Ne}(2 p), \mathrm{Ne}^{+}, \mathrm{Ne}_{2}^{*}$, and $\mathrm{Ne}_{2}^{+}$. The two radiative states of the $\mathrm{Ne}(1 s)$ manifold, $\mathrm{Ne}\left(1 s_{2}\right)$ and $\mathrm{Ne}\left(1 s_{4}\right)$ were lumped into a single radiative state denoted $\mathrm{Ne}\left(1 s_{24}\right)$. Similarly, the two metastable states of the $\mathrm{Ne}(1 s)$ manifold, $\mathrm{Ne}\left(1 s_{3}\right)$ and $\mathrm{Ne}\left(1 s_{5}\right)$ were lumped into a single metastable state denoted $\mathrm{Ne}\left(1 s_{35}\right)$. $\mathrm{Ne}\left(1 s_{24}\right)$ was assigned a radiative lifetime consistent with radiation trapping at the pressures and dimensions of interest. This produces values (10s-100s ms) which greatly exceed the lifetime of the state for collisional deactivation. Higher excited states were lumped into $\mathrm{Ne}(2 p)$ and have the characteristics of that (a)

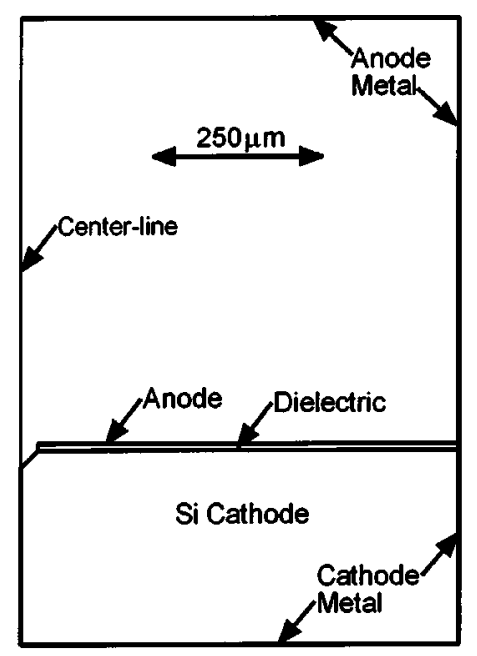

(b)

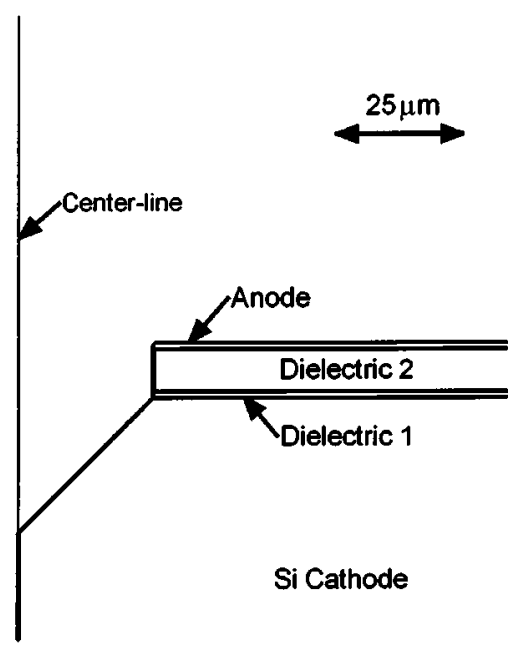

FIG. 1. Schematic of the cylindrically symmetric microdischarge geometry used in this study: (a) entire computational domain and (b) closeup of the MD cavity. The Si cathode is backed (bottom and outer radius) by negatively biased metal to which the ballast resistor is attached.

state. The $\mathrm{Ne}(2 p)$ state is radiatively coupled to $\mathrm{Ne}\left(1 s_{24}\right)$ and $\mathrm{Ne}\left(1 s_{35}\right)$ but not the ground state. Electron impact cross sections for excitation were largely taken from studies by Lin et al. ${ }^{19,20,23}$

In the following discussion reference will be made to behavior obeying Paschen's law for which breakdown voltages are a minimum at a characteristic value of $p d .^{33}$ The breakdown voltage increases at lower pressures due to the longer mean free paths reducing opportunity for ionization, and at higher pressures due to higher collisionality. Although Paschen's law strictly applies only to one-dimensional, planar discharges we will describe MD devices as obeying Paschen's law if their operating voltages obey similar scalings.

\section{PROPERTIES OF MICRODISCHARGE DEVICES}

The geometry used in this study, shown schematically in Fig. 1, was patterned after pyramidal microdischarge devices fabricated and studied by Park et al. ${ }^{1}$ The experimental device is a square pyramid which would require a threedimensional model to fully resolve. For computational expediency a cylindrically symmetric geometry was used. The 


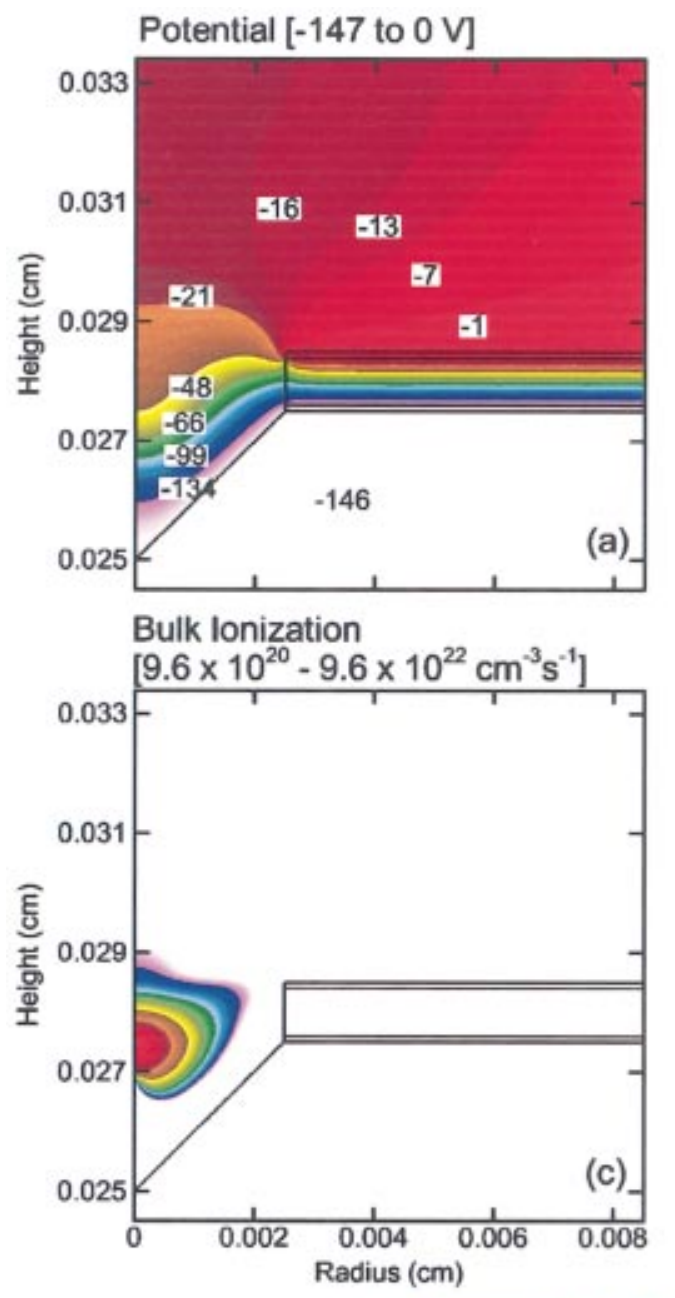

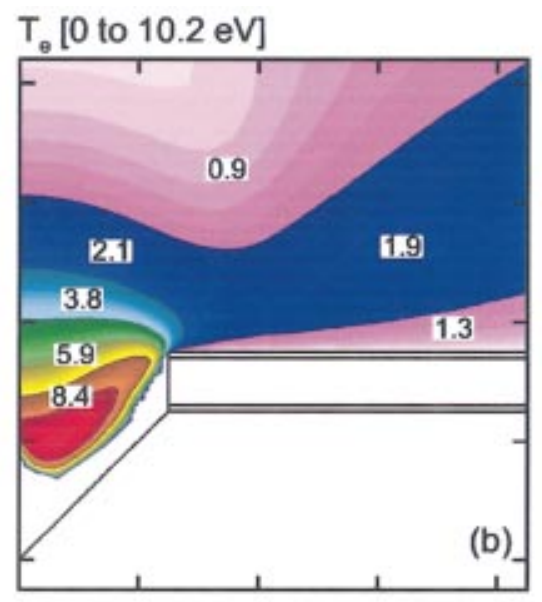

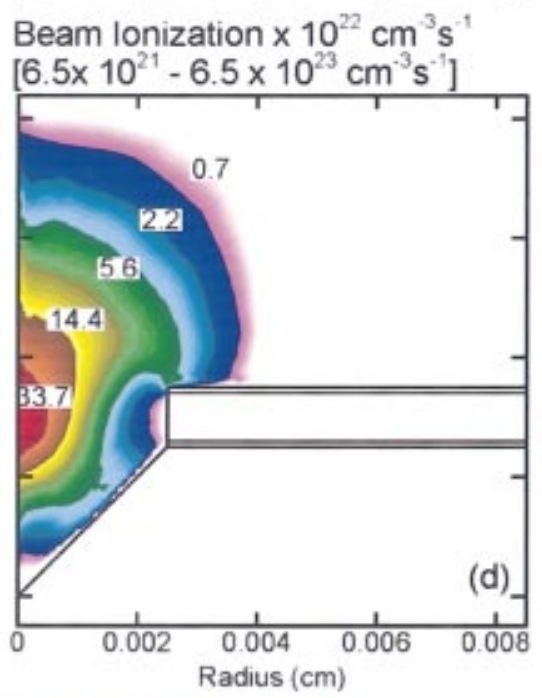

FIG. 2. (Color) Plasma characteristics for the base case (600 Torr $\mathrm{Ne}$, $-200 \mathrm{~V}, 1 \mathrm{M} \Omega$ ballast): (a) electric potential, (b) electron temperature, (c) rate of ionization by bulk electrons, and (d) rate of ionization by beam electrons (with contour labels in units of $\left.10^{22} \mathrm{~cm}^{-3} \mathrm{~s}^{-1}\right)$. The potential and electron temperature are on linear scales. The rates of ionization are plotted using a 2-decade log scale. The ranges of plotted values are noted in each figure. microdischarge cavity for the base case consists of a $25 \mu \mathrm{m}$ radius inverted cone etched into a $\mathrm{Si}$ substrate (conductivity $=0.1 / \Omega \mathrm{cm}$ ) which acts as the cathode. The outer boundaries of the Si cathode are in contact with a cathode metal. The top surface of the $\mathrm{Si}$ is covered by a $1 \mu \mathrm{m}$ thick $\mathrm{Si}_{3} \mathrm{~N}_{4}$ overlayer, $8 \mu \mathrm{m}$ thick polyminide dielectric, and a $0.2 \mu \mathrm{m} \mathrm{Ni}$ anode. The thickness of the $\mathrm{Ni}$ layer in the experiments is $0.1 \mu \mathrm{m}$. The thicker layer used here was for computational expediency. Test cases having a thinner $\mathrm{Ni}$ layer were run to confirm that our results are not prejudiced by our choice of Ni thickness. The periphery of the computational domain (except on the axis) is metal held at either the anode or cathode potential. The unstructured mesh consisted of triangles optimized to equilateral wherever possible. There are approximately 6700 total nodes with 3700 nodes in the plasma. To resolve the microdischarge while having boundaries sufficiently far away to both mimic the experimental device and not to prejudice the plasma and electrodynamics in the microdischarge region, the mesh spacing is varied from approximately $0.8 \mu \mathrm{m}$ in the inverted cone to 30 $\mu \mathrm{m}$ in the outer periphery, a dynamic range of about 40 . We treated the $\mathrm{Si}$ cathode as a conductive amorphous material and so have not considered the consequences of band bending at the surface by the electric field on electron emission.
The results discussed here are for the steady state obtained by time integration of the equations discussed in Sec. II. Before discussing those results, a few qualitative observations will be made. Starting the MD devices was problematic. Simply beginning with small electron and ion densities (e.g., $10^{5} \mathrm{~cm}^{-3}$ ) and applying voltage usually resulted in the electrons and ions being swept out of the microdischarge cavity by the large, unshielded electric field in the center of the MD device. Reliable starting required initial conditions of a significant density of a charge neutral plasma $\left(>10^{12} \mathrm{~cm}^{-3}\right.$ ) placed in the microdischarge cavity so that a portion of the applied voltage was shielded into a structure resembling a cathode fall. This high plasma density also supplied sufficient ion flux to the cathode to provide secondary electron emission to sustain the plasma. The voltage required to initiate the plasma was higher than the voltage in the steady state (e.g., -250 to $-300 \mathrm{~V}$ compared to $-200 \mathrm{~V}$ ). The lower cw voltage resulted from the buildup of excited states which enabled more efficient multistep ionization, and full formation of the cathode fall, thereby producing more energetic secondary electrons. These observations qualitatively agree with experimental practice. ${ }^{34}$

The scaling and operation of microdischarge sources is largely determined by the ability to form a cathode fall 


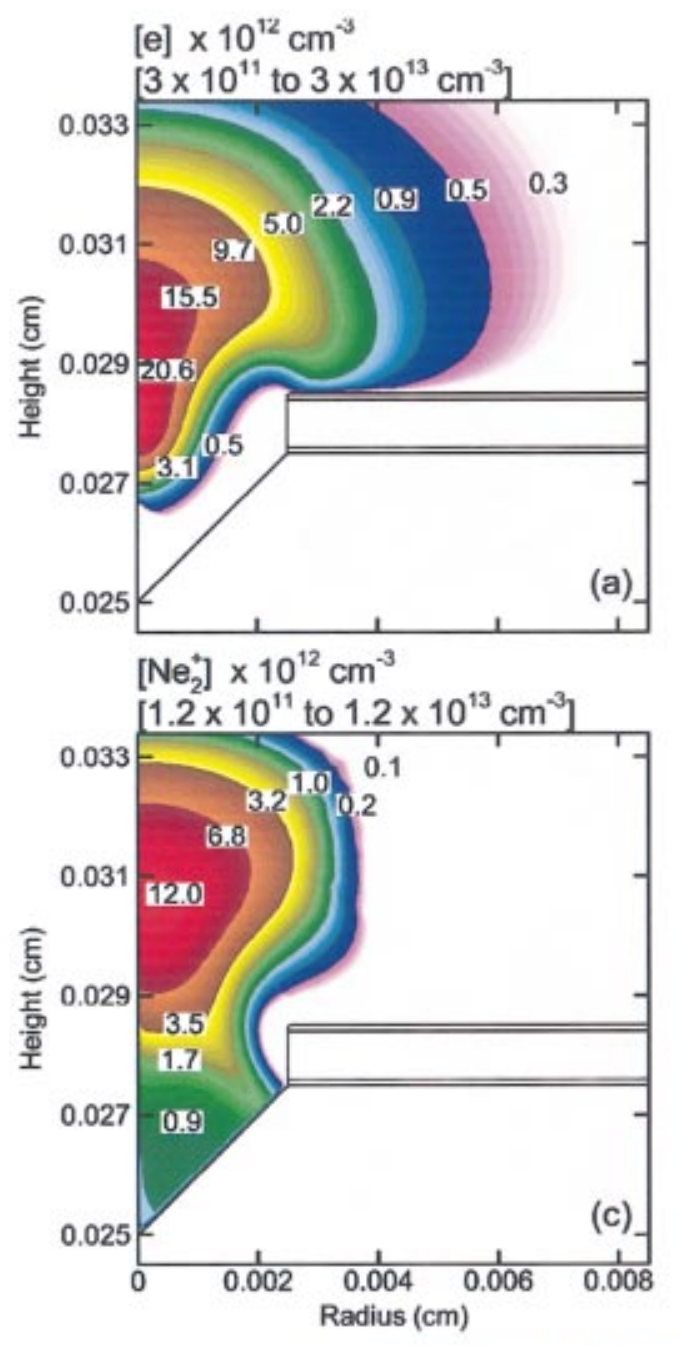

MIN
[Ne'] $\times 10^{12} \mathrm{~cm}_{3}$

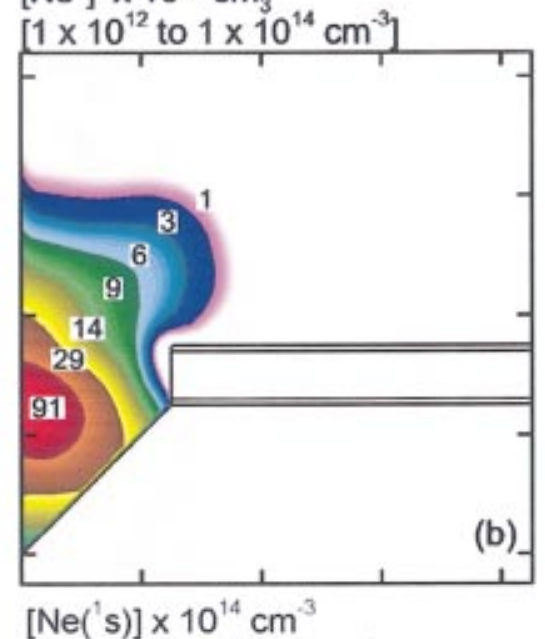

(b)

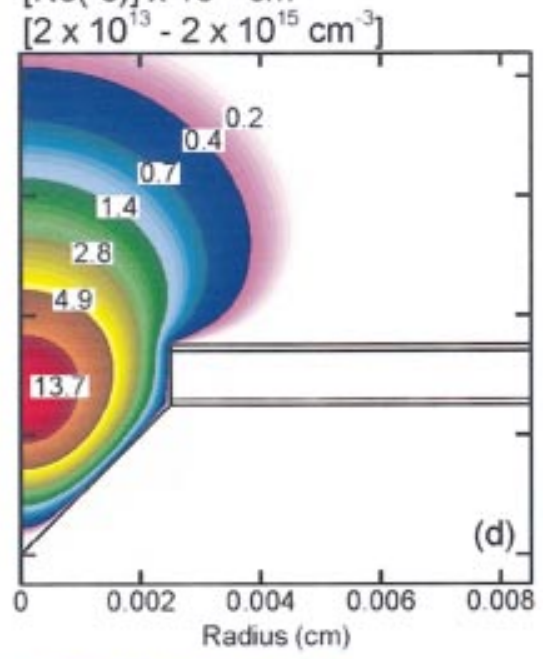

FIG. 3. (Color) Plasma characteristics for the base case (600 Torr $\mathrm{Ne}$, $-200 \mathrm{~V}, \mathrm{M} \Omega$ ballast): (a) electron density, (b) $\mathrm{Ne}^{+}$density, (c) $\mathrm{Ne}_{2}^{+}$density, and $\operatorname{Ne}\left({ }^{1} s\right)$ density. The values are plotted using a 2-decade log scale. The ranges of plotted values are noted in each figure. Contour labels are in units indicated at top of each figure. within the physical structure. (For purposes of discussion, true scaling requires that magnitudes and relative distributions of plasma properties remain the same for the same value of the scaling parameter.) In principle, all operating conditions should scale as $p d$ (pressure $\times$ characteristic dimension). However when the device dimensions shrink to be commensurate to the cathode fall thickness, then the range of operating conditions is restricted. If we assume that the ion density is constant in the cathode fall and the electron density is negligible, then the thickness of the cathode fall is $\lambda=\left[2 V_{c} \varepsilon_{0} /\left(q n_{I}\right)\right]^{1 / 2}$, where $V_{c}$ is the cathode fall voltage and $n_{I}$ is the ion density. For example, in order for a cathode fall of $-150 \mathrm{~V}$ to fit inside a microdischarge device $25 \mu \mathrm{m}$ wide, the ion density must exceed $3 \times 10^{13} \mathrm{~cm}^{-3}$.

MD devices operating in rare gases at 100 s of Torr also differ from their low pressure analogues operating at the same $p d$ (but at a few Torr) due to the importance of threebody dimerization reactions which produce molecular ions. The density of molecular ions in low pressure devices is small compared to monomer ions and the rate of electronion recombination of monomer ions is negligible. As a result, ion loss by diffusion dominates. In high pressure MD devices where the density of molecular ions may exceed that of monomer ions, volumetric recombination may exceed the rate of loss by diffusion, even in small devices. For example, for an ion mobility of $1 \mathrm{~cm}^{2} / \mathrm{V} \mathrm{s}$ (typical of near atmospheric pressure), diffusion length of $10 \mu \mathrm{m}$, electron density of $3 \times 10^{13} \mathrm{~cm}^{-3}$, ion temperature of $0.05 \mathrm{eV}$, and electron temperature of $3 \mathrm{eV}$, the rate of loss of molecular ions by recombination exceeds that by diffusion by a factor of 40 .

These trends are illustrated by predictions of plasma parameters for the base case conditions of 600 Torr $\mathrm{Ne}$ and $-200 \mathrm{~V}$ applied to the cathode through a $1 \mathrm{M} \Omega$ ballast resister. The electric potential, electron temperature, ionization by bulk electrons, and ionization by secondary beam electrons are shown in Fig. 2. The densities of electrons, $\mathrm{Ne}^{+}$, $\mathrm{Ne}_{2}^{+}$, and $\mathrm{Ne}(1 s)$ [sum of $\mathrm{Ne}\left(1 s_{24}\right)$ and $\mathrm{Ne}\left(1 s_{35}\right)$ ] are shown in Fig. 3. The voltage drop across the plasma is $-147 \mathrm{~V}$ with approximately $-97 \mathrm{~V}$ expended in the cathode fall and $-50 \mathrm{~V}$ dropped across the bulk plasma. The current is $55 \mu \mathrm{A}$ which, with a $1 \mathrm{M} \Omega$ ballast resistor, produces a resistance $2.6 \mathrm{M} \Omega$ for the plasma. These $V-I$ values compare favorably with experiments. ${ }^{1}$

The peak electron density is $3 \times 10^{13} \mathrm{~cm}^{-3}$ on axis centered on the mouth of the MD cavity. The electron density is 

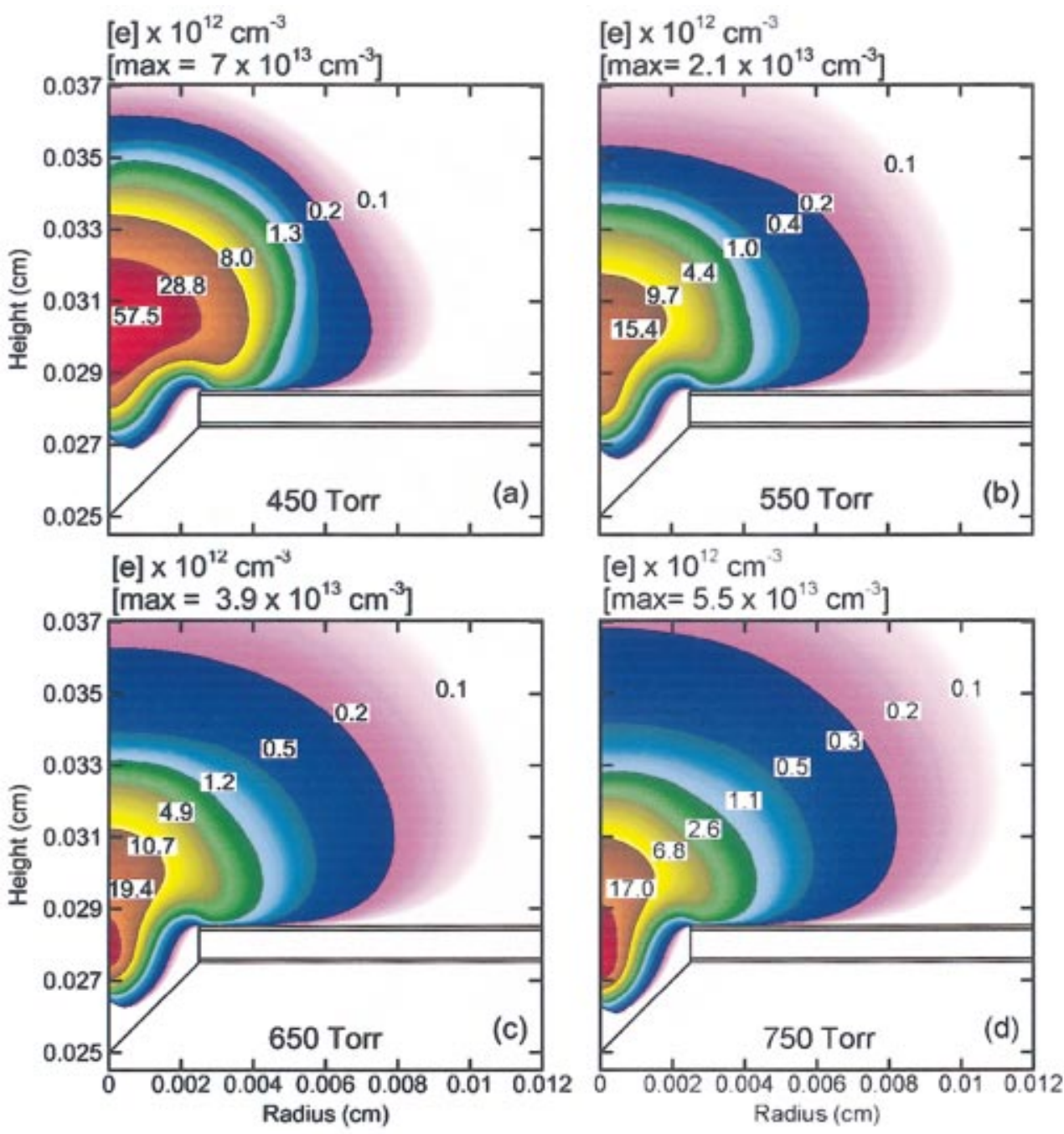

$1 \times 10^{11} \mathrm{~cm}^{-3}$

FIG. 4. (Color) Electron density for different pressures using a with $1 \mathrm{M} \Omega$ ballast resistor: (a) 450 Torr ( $-235 \mathrm{~V}$ ), (b) 550 Torr ( $-200 \mathrm{~V}$ ), (c) 650 Torr $(-200 \mathrm{~V})$, and (d) 750 Torr $(-200 \mathrm{~V})$. The values are plotted using a log scale having the range $1 \times 10^{11}-7 \times 10^{13} \mathrm{~cm}^{-3}$. Contour labels are in units of $10^{12} \mathrm{~cm}^{-3}$. The devices operating at higher pressures produce more confined plasmas.

produced by ionization sources by bulk electrons peaking at $1 \times 10^{23} \mathrm{~cm}^{-3} \mathrm{~s}^{-1}$ and by beam electrons peaking at $7 \times 10^{23} \mathrm{~cm}^{-3} \mathrm{~s}^{-1}$. The ionization by bulk electrons is sustained in part by an electron temperature which peaks at 10 $\mathrm{eV}$ in the high electric field at the edge of the presheath, a region populated by a small number of electrons. These hot electrons originate in low energy secondary electrons produced by the electron beam which are in turn accelerated by the cathode fall. The majority of the ionization by bulk electrons results from collisions with $\mathrm{Ne}(1 s)$, whose density peaks at $2 \times 10^{15} \mathrm{~cm}^{-3}$ in the same volume. The swath of higher electron temperature $(\approx 2 \mathrm{eV})$ outside the mouth of the MD results from electrons coming into equilibrium with the larger $E / N$ near the anode. This is not particularly meaningful since the electron density in those regions is small.

The total ionization is dominated by beam electrons, both in terms of the peak ionization source and the volume integrated source. The beam ionization peaks on axis in the throat of the MD cavity where the beams from the moderately opposing cathodes converge. The mean free path for energy loss for electrons having gained an energy equal to the cathode fall is a few microns, enabling penetration into the MD cavity. As electric field lines loop back to the anode, there is some concentration of the secondary beam as it slows, converging on the edge of the anode.

The cathode fall thickness is $\approx 5 \mu \mathrm{m}$ and is nearly devoid of electrons. The ion density, peaking at nearly $10^{14} \mathrm{~cm}^{-3}$, dominates the cathode fall with the maximum ion flux being incident near the center of the bevel. This, in turn, causes the secondary electron beam to be launched from that location. The ion density is clearly differentiated into regions dominated by $\mathrm{Ne}^{+}$or $\mathrm{Ne}_{2}^{+}$. Monomer ions which are produced in regions of high electric field drift into the cathode before they dimerize to form $\mathrm{Ne}_{2}^{+}$. Therefore, the cathode fall is largely dominated by $\mathrm{Ne}^{+}$. In the periphery of the plasma, where diffusion of ions moves them into regions of low electric field, the ion density is dominated by $\mathrm{Ne}_{2}^{+}$as there is sufficient time for dimerization to occur before the monomer is lost to recombination or diffusion. As 
the lifetime of $\mathrm{Ne}_{2}^{+}$for dissociative recombination is $\approx 1 \mu \mathrm{s}$ where its density peaks, the population of $\mathrm{Ne}_{2}^{+}$is rapidly depleted. There are few regions within the MD cavity where a truly quasineutral plasma is produced. With Debye lengths of a few to $10 \mu \mathrm{m}$, commensurate with MD dimensions, this is not an unexpected result.

Comparison of the behavior of the MD devices as a function of pressure while keeping other parameters constant is somewhat complicated by Paschen curve considerations. For devices whose ionization is dominated by cathode fall phenomena, operating voltages should resemble those for breakdown. For example, at sufficiently low pressure the MDs are on the low pressure side of Paschen's curve and require a higher applied voltage to sustain (or a smaller ballast resistor). The lowest pressure that would sustain with $-200 \mathrm{~V}$ and $1 \mathrm{M} \Omega$ ballasting is about 550 Torr, requiring somewhat linear increases in voltage with decreasing pressure to $-235 \mathrm{~V}$ at 450 Torr. Electron densities for pressures from 450 to 750 Torr for the same ballasting (1 M $\Omega$ ) are shown in Fig. 4. The cases for pressures of 550-750 Torr are for voltages of $-200 \mathrm{~V}$, whereas the case at 450 Torr has a voltage of $-235 \mathrm{~V}$. As the pressure increases, the plasma is more confined to the MD cavity as the penetration of the beam electrons decreases and is efficiently stopped within the cavity while diffusion losses decrease. For these reasons, when the voltage is held constant, the electron densities increase with increasing pressure in spite of a somewhat larger rate of recombination due to the larger density of molecular ions. The increase in plasma density produces a decrease in sheath thickness. At 750 Torr, the plasma is largely contained within the MD cavity whereas at 450 Torr, the plasma is more diffusive and largely sits above the throat to the MD cavity.

These trends are illustrated by the peak electron densities as a function of pressure (450-800 Torr) which are shown in Fig. 5(a). For cases where the applied voltage is constant at $-200 \mathrm{~V}$, the peak electron density linearly increases with pressure, from $2 \times 10^{13} \mathrm{~cm}^{-3}$ at 550 Torr to $7 \times 10^{13} \mathrm{~cm}^{-3}$ at 800 Torr. These trends are similar to those observed by Penache et al. ${ }^{35}$ who measured a nearly linear increase in electron density with increasing pressure, albeit for different operating conditions. The comparison to discharges at lower pressures is complicated by their having to operate at higher voltages. The increase in operating voltage produces an increase in peak plasma density with decreasing pressure over the range investigated. This range of operating conditions appears to bracket the minimum in discharge voltage as one would find in a discharge obeying Paschen's law. For example, the voltage drop across the plasma as a function of pressure is shown in Fig. 5(b) for a ballast resistor of $1 \mathrm{M} \Omega$. The minimum in sustaining voltage is approximately at 700 Torr, with significant increases towards lower pressure. These results are, to some degree, a function of the combination of applied voltage and ballast resistor which is used. These values ultimately determine the current density which in turn control the excited state density and cathode fall thickness. Higher excited state densities with their higher ionization rates, and thinner cathode falls with more ener-
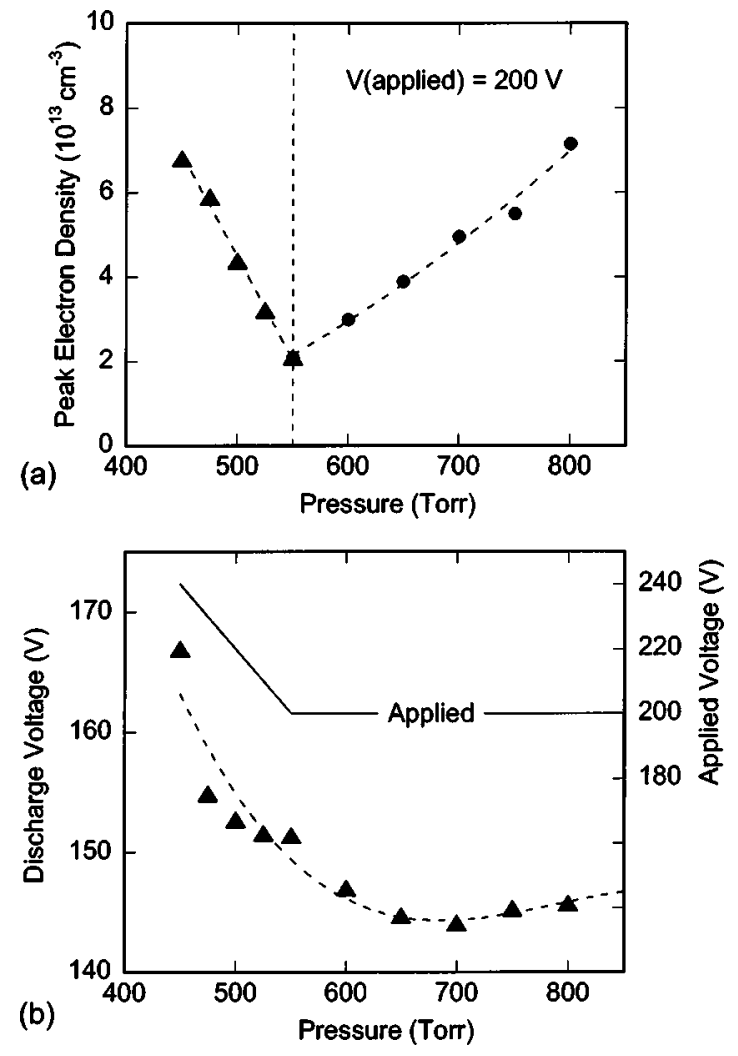

FIG. 5. Plasma characteristics as a function of pressure for MD devices operating in neon: (a) peak electron density and (b) discharge and applied voltage. At the constant voltage of $-200 \mathrm{~V}$, the peak electron density increases nearly linearly with pressure. Due to Paschen behavior, discharges could not be sustained at $-200 \mathrm{~V}$ at less than 550 Torr. The discharge voltage has a minimum near 700 Torr.

getic (less collisional) beam electrons, shift the minimum in the discharge voltage to lower pressures.

Power deposition by electrons in the bulk plasma for the base case peaks at many $100 \mathrm{~s} \mathrm{~kW} / \mathrm{cm}^{3}$ in the middle of the MD cavity. The proportion of this power which directly results in gas heating through charge exchange or ion transport is small, producing a temperature rise of only $20-50 \mathrm{~K}$ above ambient. This small temperature rise and subsequently small rarefaction is mediated by the close proximity and high thermal conductivity of the substrate which efficiently cools the gas, and the small volume of the directly heated gas. The dissipation of heat by thermal conductivity into the larger volume of the chamber efficiently regulates the temperature of the gas in the MD cavity. In spite of relatively small temperature excursions, there are, however, measurable differences in MD performance. For example, when including gas heating and rarefaction, the required voltages are higher when operation is on the low pressure side of Paschen's curve as the rarefaction shifts the MD to smaller values of $p d$.

Gas heating in MD devices is highly dependent on gas composition and geometry. The smaller dimensions of MD devices compared to conventional low pressure devices produces a larger surface-to-volume ratio, thereby providing a higher degree of regulation of gas temperature by thermal conduction. Nevertheless, high gas temperature excursions may occur in MD devices. For example, Penache et al. ${ }^{35}$ 
measured gas temperatures as high as $1100 \mathrm{~K}$ in MD devices sustained in Ar at 300 Torr. These higher temperatures likely resulted from their operating at higher plasma densities $\left(5 \times 10^{15} \mathrm{~cm}^{-3}\right)$ and larger dimensions $(300 \mu \mathrm{m})$ producing a higher rate of heating and lower rate of cooling by thermal conduction.

The onset of MD behavior resembling a hollow cathode (or negative glow) discharge at lower pressures or higher operating voltages has been experimentally characterized by the appearance of optical emission from high lying states of the rare gas ion. ${ }^{1}$ We are not tracking these specific states in the model. To characterize the onset of behavior resembling hollow cathode (or negative glow) discharges we used the ratio $\alpha=S\left(\mathrm{Ne}^{2+}\right) /\left[S\left(\mathrm{Ne}^{+}\right)+S\left(\mathrm{Ne}^{2+}\right)\right]$. This is the ratio of the total rate of electron impact ionization of $\mathrm{Ne}$ from the ground state forming $\mathrm{Ne}^{2+}$ to the total rate of ionization from the ground state forming both $\mathrm{Ne}^{2+}$ and $\mathrm{Ne}^{+}$. Ionization directly producing $\mathrm{Ne}^{2+}$ (threshold $41 \mathrm{eV}$ ) is most sensitive to the tail of the electron energy distribution and to the beam electrons. Ionization directly producing $\mathrm{Ne}^{+}$(threshold $21 \mathrm{eV}$ ) is, in comparison, more sensitive to the bulk of the electron energy distribution. Large values of $\alpha$ signify that excitation and ionization are dominated by the beam component which is characteristic of discharges resembling hollow cathode or negative glow devices.

$\alpha$ is shown in Fig. 6 as a function of position for 450 and 800 Torr. The ratio is also shown for two locations [on axis at the throat of the MD (height $=0.028 \mathrm{~cm}$ ) and downstream (height $=0.0305 \mathrm{~cm}$ ) ] as a function of pressure. At 800 Torr $\alpha$ is small (negligible with values dominated by numerical noise in the Monte Carlo simulation) throughout the MD cavity. Appreciable double ionization occurs only near the tip of the anode and along the axis. The former results from electric field enhancement and a convergence of beam electrons. The latter results from there being a small directed component of the beam electrons along the axis. (Note that the on-axis peak is likely exaggerated by statistical noise in the simulation but it is nevertheless persistent.) At 450 Torr, $\alpha$ is large (above 0.1) throughout the MD cavity and as well as downstream. This largely results from the beam electrons penetrating further into the bulk plasma with the higher operating voltage at the lower pressure. $\alpha$ is large downstream because the normalized electric field $\left(E / N<5-6 \times 10^{-17} \mathrm{~V} \mathrm{~cm}^{2}\right.$, or 5-6 Td) and electron temperature $(<2 \mathrm{eV})$ are both sufficiently low that the rate of ionization by bulk electrons is small. As a result, ionization is dominated by beam electrons. $\alpha$ increases above the noise level at the mouth of the MD cavity as the pressure falls below 600 Torr, and at the downstream location at lower pressures. Based on this diagnostic, we would characterize the MDs as operating in a hollow cathode (or negative glow) mode at pressures of $\$ 550$ Torr and in a Townsend mode at higher pressures. The pressure (or $p d$ ) at which experimental devices undergo this transition depends critically on the details of their geometry. ${ }^{1}$ For example, replacing the annular anode with a screen anode which covers the mouth of the MD cavity greatly increases the excitation of high lying states at a given pressure, most likely due to the higher electric fields which accelerate beam electrons within the cavity.

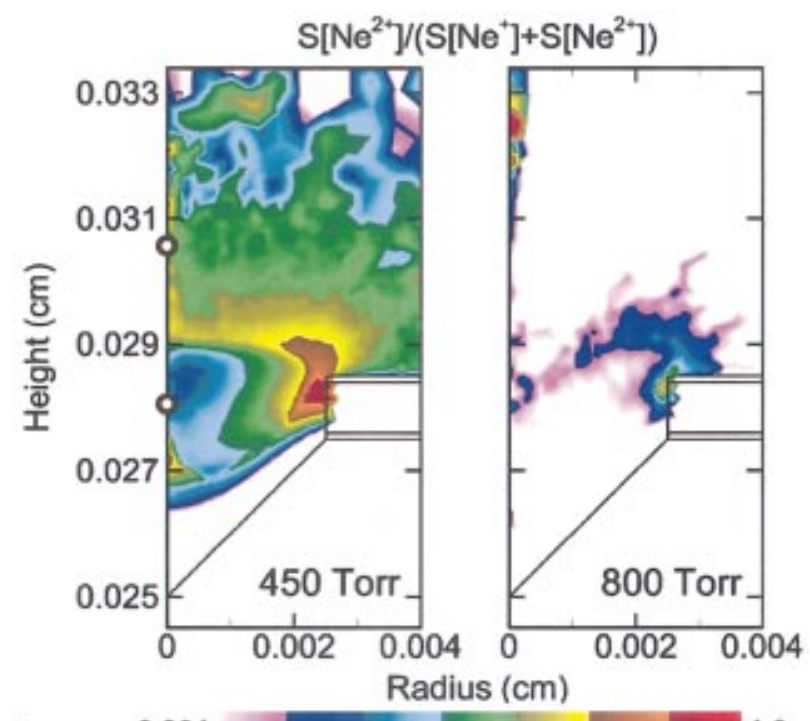

(a)
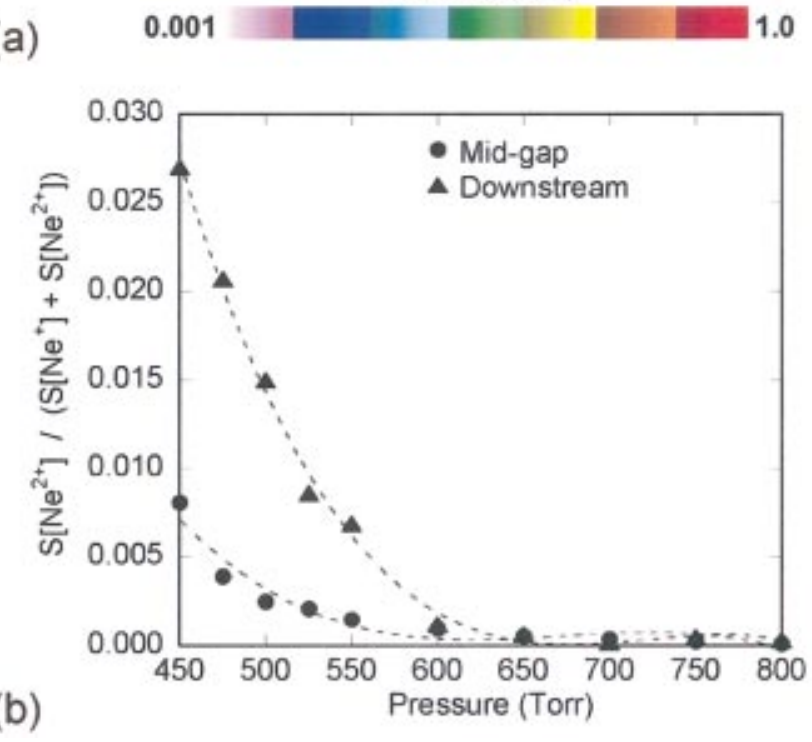

FIG. 6. (Color) Ratio of ionization rates characterizing the hollow cathode (negative glow) nature of the microdischarge device. The quantity plotted is $\alpha$, the ratio of the total electron impact rate (bulk and beam electrons) for double ionization of $\mathrm{Ne}$ from the ground state to the total ionization from the ground state $\left(\mathrm{Ne}^{2+}\right.$ and $\mathrm{Ne}^{+}$): (a) $\alpha$ as a function of position for 450 Torr and 800 Torr plotted on a 3 decade $\log$ scale and (b) $\alpha$ as a function of pressure on axis at mid gap (height $=0.028 \mathrm{~cm}$ ) and downstream (height $=0.0305 \mathrm{~cm}$ ). Behavior resembling hollow cathode or negative glow discharges, indicated by large values of $\alpha$, occur at pressures of $<600$ Torr.

Experimentally observed transitions for similar MD devices occur at 500-700 Torr.

Direct comparisons of voltage-current curves with experimental devices is difficult due to their sensitivity on device structures, secondary electron emission coefficients, choice of ballast resistor and material properties. For example, both positive and negative differential resistances can be obtained in the same device depending on the range of currents and voltages. ${ }^{1}$ The experimental devices are fully three-dimensional, pyramidal structures whereas the devices modeled here are two-dimensional conical representations. Nevertheless, favorable comparisons to experiments have been made. For reference, experimental devices operating in neon with similar dimensions for pressures of 500-700 Torr 


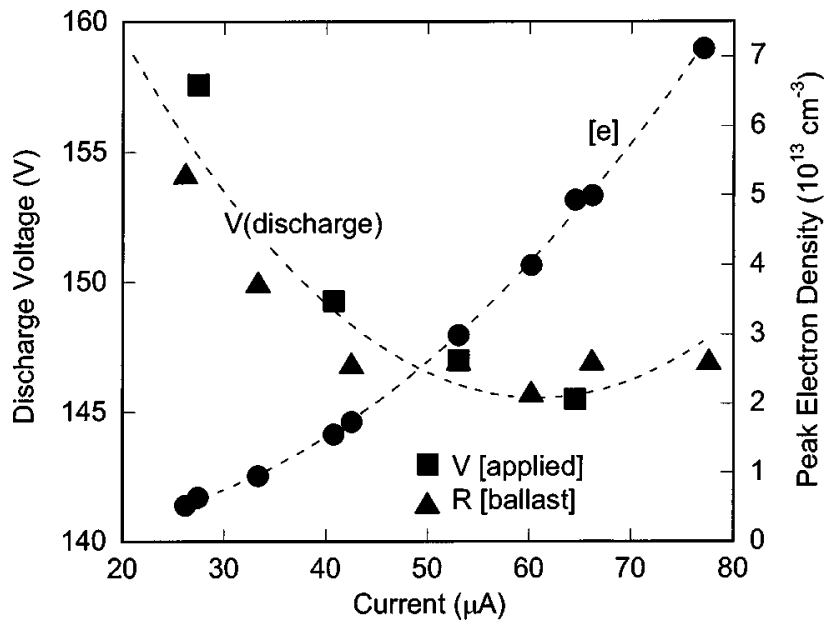

FIG. 7. Discharge voltage and peak electron density as a function of discharge current for MD sustained in 600 Torr Ne. The $I-V$ characteristics were produced by either varying applied voltage (with a $1 \mathrm{M} \Omega$ ballast resistor) or varying the value of the ballast resistor (with $-200 \mathrm{~V}$ applied). These devices display negative differential resistance at low current and indications of positive differential current at high current density.

draw currents of $20-35 \mu \mathrm{A}$ with discharge voltages of $160-$ $195 \mathrm{~V}$ with a $1 \mathrm{M} \Omega$ ballast. $^{1,34}$

Current-voltage $(I-V)$ characteristics obtained from the model for 600 Torr are shown in Fig. 7 along with the peak electron density. The applied voltage and ballast resistor were separately varied while keeping the other constant to generate the $I-V$ characteristic. The peak electron density is linearly proportional to current with a change in slope at higher currents, perhaps indicating a transition from a normal glow to an abnormal glow. For these conditions we obtained a total of currents $20-80 \mu \mathrm{A}$ with a negative differential resistance at low currents and indications of positive differential resistance at high currents. These results imply that low currents are not limited by secondary processes at the cathode and multistep ionization, which scales with current density, and is an important source of ionization. The positive differential resistance at higher currents for experimental devices in similar operating regimes reflects the need for additional ionization by beam electrons or a secondary emission process which is sensitive to the electric field in the cathode fall.

The sensitivity of the performance of MD devices to small changes in operating voltage and ballast resistance, which ultimately determine current density, is in part explained by the cathode fall thickness being commensurate with device dimensions. For example, closeups of the electron density, plasma potential, and ionization source by beam electrons are shown in Fig. 8 for devices operating a low current density $(-200 \mathrm{~V}, 1.75 \mathrm{M} \Omega$ ) producing a low peak electron density $\left(5.3 \times 10^{12} \mathrm{~cm}^{-3}\right)$ and high current density $(-210 \mathrm{~V}, 1 \mathrm{M} \Omega)$ producing a high peak electron density $\left(4.9 \times 10^{13} \mathrm{~cm}^{-3}\right)$. In the low current density case, the charge density in the cathode fall is low and so the cathode fall thickness is larger than the high current density case. The electric field in the cathode fall is therefore smaller and extends over a larger number of mean free paths for the beam electrons. The end result is that at the lower current density, beam electrons are more collisional during their acceleration in the cathode fall, reach a lower peak energy, and produce a more distributed but smaller ionization source. At the higher current density, the cathode fall is thinner and electric field larger. Beam electrons reach higher peak energies, and the ionization source is more concentrated in the bulk plasma.

The development of microdischarge sources has been motivated by $p d$ scaling; devices having small dimensions should operate similarly to macroscopic devices provided the product of pressure and characteristic dimension is kept constant. In the context of multidimensional devices, similar operation means having approximately the same spatial distribution of plasma and excited state densities when normalized by $d$. As previously discussed, this scaling is less straightforward for MD devices having complex geometries or when the sizes of the devices are commensurate with their cathode fall thickness. The $p d$ scaling of the pyramidal geometry was investigated by keeping $p d=1.5$ Torr $\mathrm{cm}$ as in the base case device while using the radius (and depth) of the MD cavity as the characteristic dimension. The thickness of the dielectric was held constant since in experimental devices a critical thickness is required to prevent surface arcing. The peak electron densities were kept approximately the same by adjusting the value of the ballast resistor with a constant applied voltage of $-200 \mathrm{~V}$ as in the base case. The electron densities for a $15 \mu \mathrm{m}$ device operating at 1000 Torr (ballast $2.1 \mathrm{M} \Omega$ ), $25 \mu \mathrm{m}$ device operating at 600 Torr (base case, ballast $1 \mathrm{M} \Omega$ ), and a $37.5 \mu \mathrm{m}$ device operating a 400 Torr (ballast $285 \mathrm{k} \Omega$ ) are shown in Fig. 9.

The peak electron densities are essentially the same for all cases $\left(2.96,2.99\right.$, and $2.96 \times 10^{13} \mathrm{~cm}^{-3}$ for the 15,25 , and $37.5 \mu \mathrm{m}$ MDs) while the total currents, 29, 53, and 88 $\mu \mathrm{A}$, correspond to average current densities of $1.5,1.0$, and 0.75 relative to the base case. The electron density in all cases peaks at approximately the mid-height of the dielectric where the converging $e$-beam component is most constricted and the ionization rate is the largest. The qualitative distributions of the plasma, when distances are normalized by pressure, are similar though not identical. The centroid of the plasma becomes progressively more confined to the MD cavity as the pressure increases and $d$ decreases, whereas the extent of the plasma is greater. This trend is demonstrated by Fig. 9(d) where the electron density is shown as a function of normalized height at $r=0$. The expectation is that the more rapid rate of three-body dimerization at higher pressures will produce more molecular ions (e.g., $\mathrm{Ne}_{2}^{+}$) which would increase the rate of recombination. More rapid production of excited dimers (e.g., $\mathrm{Ne}_{2}^{*}$ ) should increase the rate of loss of excited states by radiation, and so the higher pressure plasmas should, on the average, be more confined. The increase in loss by diffusion at lower pressures appears to dominate the increase in recombination at higher pressures to limit the extent of the plasma. The increased confinement of the peak density at higher pressures may be attributable to the constant thickness of the insulator being a larger fraction of the MD cavity depth as the characteristic dimension shrinks. These generally favorable similarities suggest that $p d$ scaling of this geometry can be maintained to cavity sizes of 

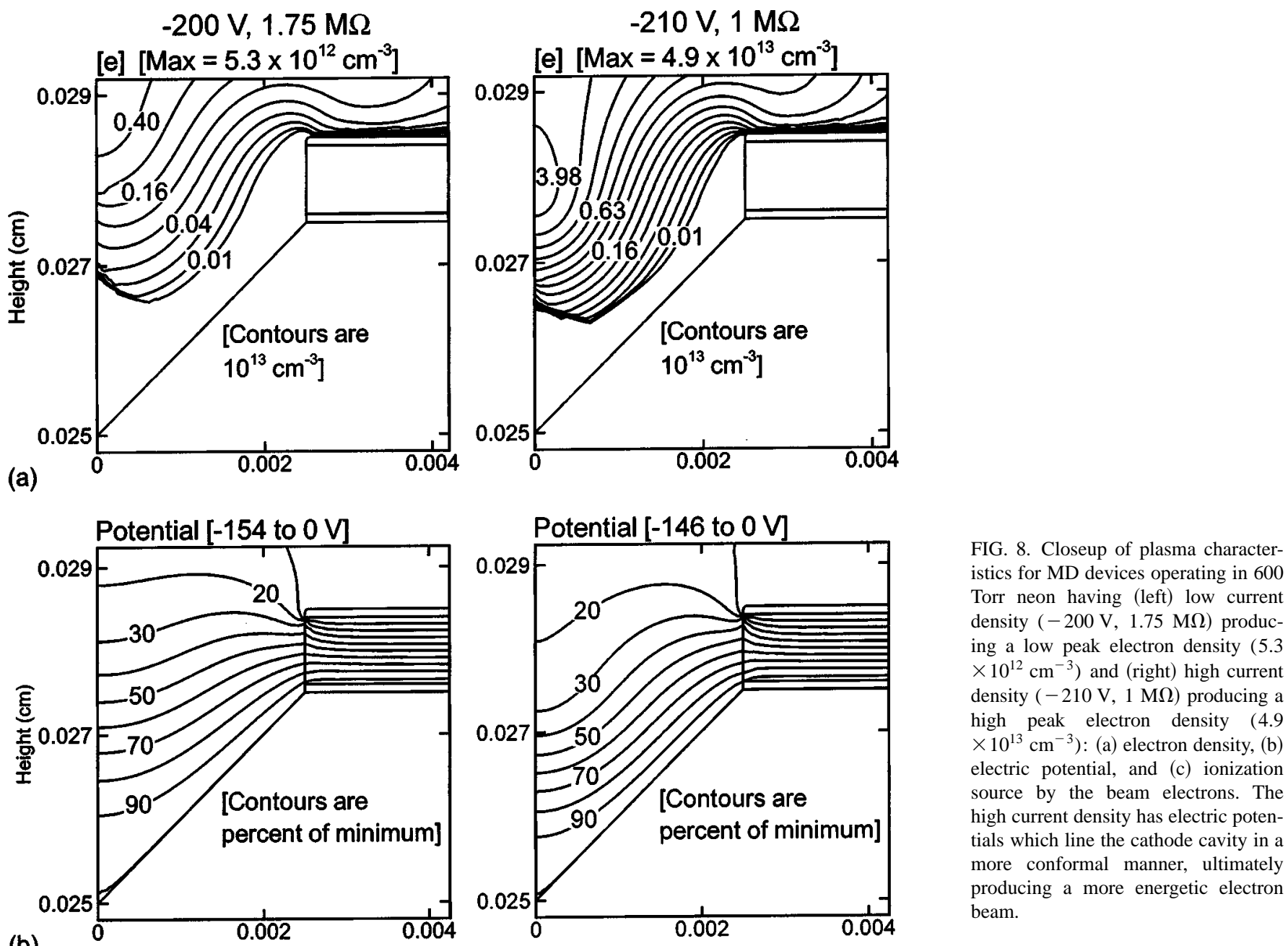

(b)
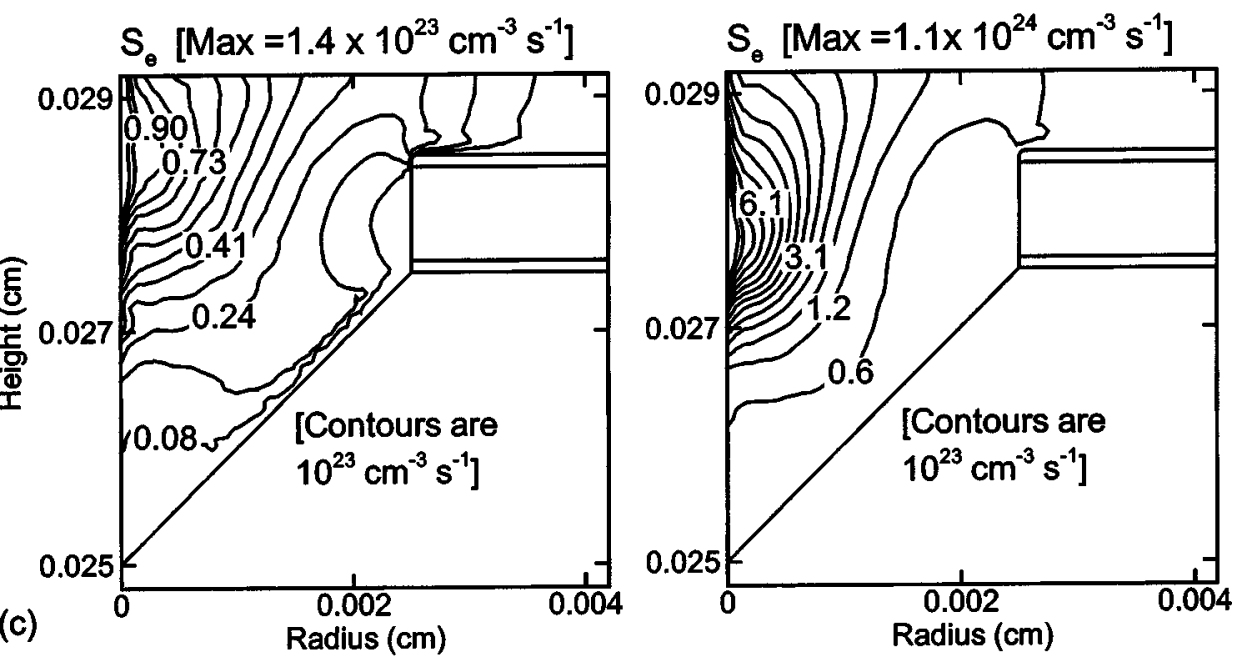

$<15 \mu \mathrm{m}$ provided that the cathode fall thickness remains a small fraction of $d$.

Maintaining the electron and current densities approximately equal is important to achieving $p d$ scaling. These trends are as illustrated by the electron densities shown in Fig. 10 for the 15 and $37.5 \mu \mathrm{m}$ MDs when the ballast resistor was kept constant at $1 \mathrm{M} \Omega$. The resulting lower current density for the $37.5 \mu \mathrm{m}$ MD (0.24 relative to the base case) produced a peak electron density of only $3.3 \times 10^{12} \mathrm{~cm}^{-3}$.
The plasma detaches from the contours of the MD cavity and sets above the mouth of the device, a situation resulting from the cathode fall being unable to fit within the confines of the MD cavity. The higher current density for the $15 \mu \mathrm{m}$ MD (3.1 relative to the base case) produced a peak electron density of $1.7 \times 10^{14} \mathrm{~cm}^{-3}$. The peak of the plasma sits deeper inside the MD cavity, a consequence of the thinner cathode fall. These results motivate the use of a somewhat modified $p d$ scaling law for nonplanar, hollow MD devices which in- 

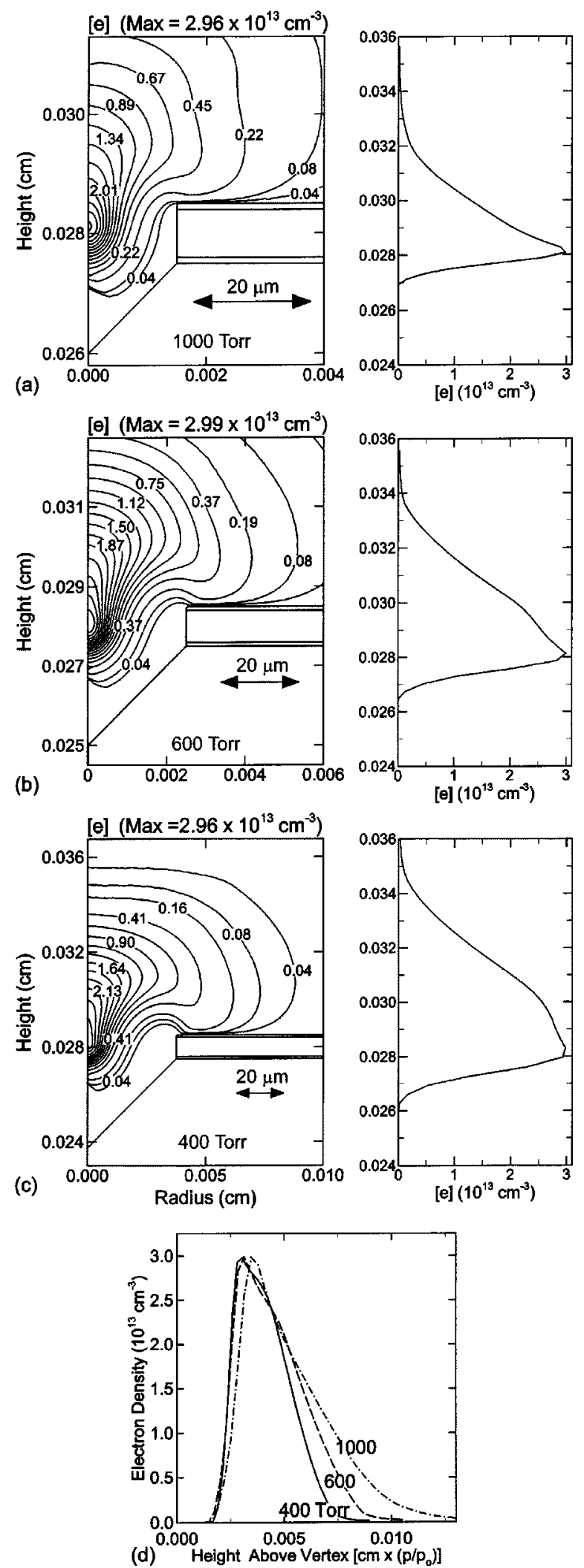

FIG. 9. Electron density in devices maintaining $p d$ and current density constant for an applied potential of $-200 \mathrm{~V}$. MD radii and pressure are: (a) $15 \mu \mathrm{m}, 1000$ Torr; (b) $25 \mu \mathrm{m}, 600$ Torr; and (c) $37.5 \mu \mathrm{m}, 400$ Torr. The line plots to the right of the contours show electron density along the $r=0$ axis. (d) Electron density along the $r=0$ axis for all three cases. The height coordinate begins at the vertex of the MD cavity and is normalized by pressure ( $p_{o}=600$ Torr). Contour labels have units of $10^{13} \mathrm{~cm}^{-3}$ and the peak electron density is noted in each frame. $p d$ scaling qualitatively holds based on similar morphology and peak density.
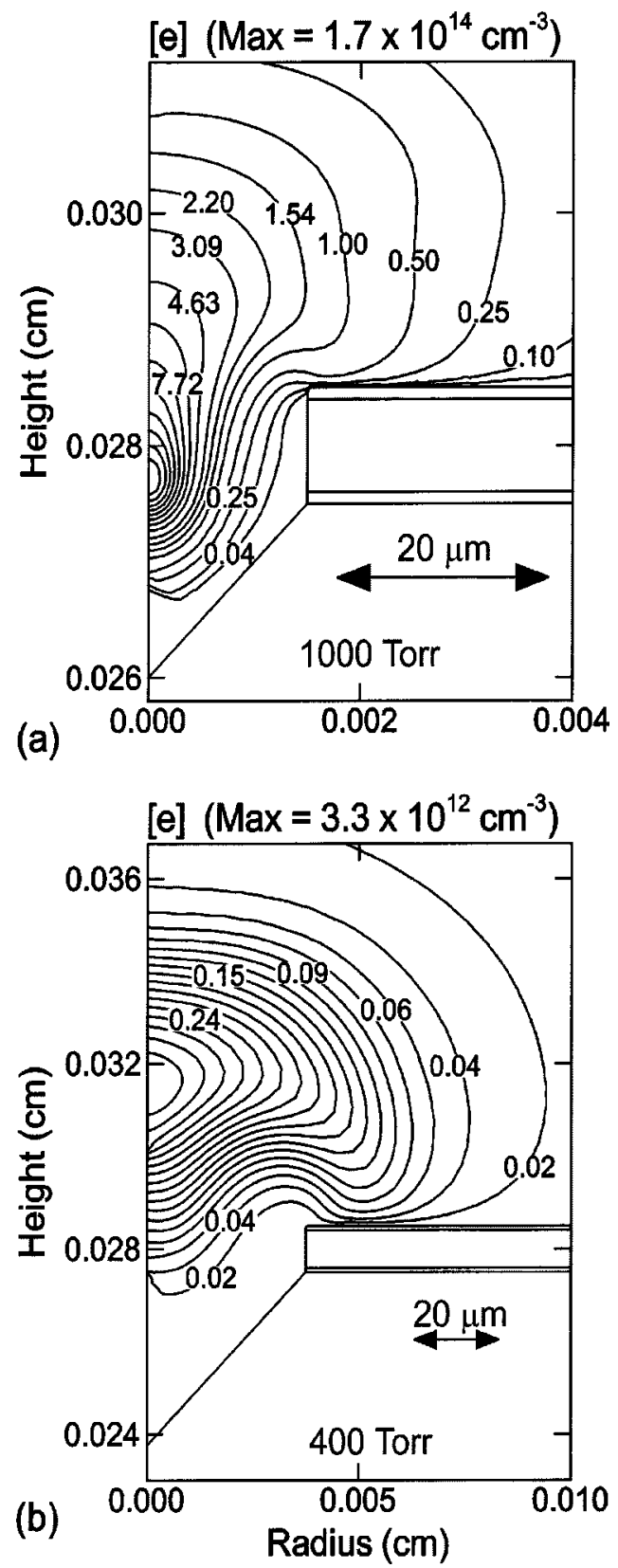

FIG. 10. Electron density in devices maintaining $p d$ and the ballast resistor constant for an applied potential of $-200 \mathrm{~V}$. MD radii and pressure are: (a) $15 \mu \mathrm{m}, 1000$ Torr and (b) $37.5 \mu \mathrm{m}, 400$ Torr. Contour labels have units of $10^{13} \mathrm{~cm}^{-3}$ and the peak electron density is noted in each frame. The lower current density of the larger MD device produces a plasma which sits atop the MD cavity. The higher current density of the smaller MD device is confined deep inside the cavity.

cludes the requirement that the cathode fall thickness as a fraction of the characteristic dimension remain constant or be smaller than a critical value. In this regard $p d \cdot(\lambda / d)$ may be an appropriate scaling law, where $(\lambda / d)$ remains constant or less than a critically small value.

The importance of three-body reactions in pressure scaling of these MD devices was investigated by performing a set of parameterizations over pressure (400-1000 Torr) where the density of the stabilizing third body was held constant at its value for 600 Torr. The $I-V$ characteristics were only nominally affected over this range. The spatial distribu- 
tion densities of radiators (e.g., $\mathrm{Ne}_{2}^{*}$ as opposed to $\mathrm{Ne}^{*}$ ) qualitatively shifted in favor of the molecular radiators at lower pressure and the atomic radiators at higher pressure.

\section{CONCLUDING REMARKS}

The operation of pyramidal microdischarge devices having dimensions of $15-40 \mu \mathrm{m}$ operating in neon at pressures of 450-1000 Torr were investigated using results from a two-dimensional plasma hydrodynamics model augmented by an electron Monte Carlo simulation for beam electrons. Behavior resembling Paschen's law for breakdown was observed with a minimum in discharge voltage occurring near 700 Torr and with increasing applied voltage being required to operate the MDs at lower pressures. The onset of behavior resembling negative glow discharges, as indicated by excitation of high lying states, occurs with decreasing pressure due to an extension of the cathode fall accelerated beam electrons into the bulk plasma. For the geometry investigated, this transition occurred at 550-600 Torr. For constant applied voltage, peak electron densities increased with increasing pressure as the beam electrons are slowed in more confined regions. When the cathode fall and Debye lengths are commensurate with the size of the MD cavity, small changes in operating parameters can produce significant variation in plasma parameters due to perturbation of the trajectories of cathode-accelerated beam electrons. By keeping $p d$ and current density constant, MD devices having similar magnitudes and spatial distributions of plasma and excited state densities can likely be obtained to dimensions of $<15 \mu \mathrm{m}$ provided the cathode fall thickness is small compared to the characteristic dimension.

\section{ACKNOWLEDGMENTS}

This work was supported by the National Science Foundation (Grants No. CTS99-74962 and CTS03-15353), Electric Power Research Institute and General Electric Research and Development Center. The author acknowledges Professor J. Gary Eden for his expert commentary and advice.

${ }^{1}$ S.-J. Park, J. Chen, C. J. Wagner, N. P. Ostrom, C. Liu, and J. G. Eden, IEEE J. Sel. Top. Quantum Electron 8, 387 (2002).

${ }^{2}$ J. Chen, S.-J. Park, Z. Fan, J. G. Eden, and C. Liu, J. Microelectromech. Syst. 11, 536 (2002).
${ }^{3}$ S.-J. Park, C. J. Wagner, and J. G. Eden, IEEE Photonics Technol. Lett. 12, 61 (2001).

${ }^{4}$ S. J. Park, J. G. Eden, J. Chen, C. Liu, and J. J. Ewing, Opt. Lett. 26, 1773 (2001).

${ }^{5}$ J. W. Frame, D. J. Wheeler, T. A. DeTemple, and J. G. Eden, Appl. Phys. Lett. 71, 1165 (1997).

${ }^{6}$ R. H. Stark and K. H. Schoenbach, Appl. Phys. Lett. 74, 3770 (1999).

${ }^{7}$ K. H. Schoenbach, A. El-Habachi, W. Shi, and M. Ciocca, Plasma Sources Sci. Technol. 6, 468 (1997).

${ }^{8}$ A. El-Habachi, W. Shi, M. Moselhy, R. H. Stark, and K. H. Schoenbach, J. Appl. Phys. 88, 3220 (2000).

${ }^{9}$ L. D. Biborosch, O. Bilwatsch, S. Ish-Shalom, E. Dewald, W. Ernst, and K. Frank, Appl. Phys. Lett. 75, 3926 (1999).

${ }^{10}$ O. B. Postel and M. A. Capelli, J. Appl. Phys. 89, 4719 (2001).

${ }^{11}$ J. B. Boeuf, J. Phys. D 36, R53 (2003).

${ }^{12}$ B. Lay, R. S. Moss, S. Rauf, and M. J. Kushner, Plasma Sources Sci. Technol. 12, 8 (2003).

${ }^{13}$ Skyblue Systems, Inc., P. O. Box 14223, Albany, NY 12212-4223 〈http:// www.skybluesystems.com〉.

${ }^{14}$ D. L. Scharfetter and H. K. Gummel, IEEE Trans. Electron Devices ED16, 64 (1969).

${ }^{15}$ SLAP Sparse Matrix Library 〈http://www.netlib.org

${ }^{16}$ M. K. Seager, Lawrence Livermore National Laboratory Technical Rep. No. UCRL-100195, 1998 〈http://www.llnl.gov〉

${ }^{17}$ R. Kinder and M. J. Kushner, J. Appl. Phys. 90, 3699 (2001).

${ }^{18}$ M. Hayashi, Nagoya Institute of Technology, Rep. No. IPPJ-AM-19, 1982.

${ }^{19}$ M. H. Phillips, L. W. Anderson, and C. C. Lin, Phys. Rev. A 32, 2117 (1985).

${ }^{20}$ J. E. Chilton, M. D. Stewart, Jr., and C. C. Lin, Phys. Rev. A 61, 052708 (2000).

${ }^{21}$ E. Krishnakumar and S. K. Srivastava, J. Phys. B 21, 1055 (1988).

${ }^{22}$ J. B. Boffard, M. L. Keeler, G. A. Piech, L. W. Anderson, and C. C. Lin, Phys. Rev. A 64, 032708 (2001).

${ }^{23}$ M. Johnson, K. Fujii, J. Nickel, and S. Trajmar, J. Phys. B 29, 531 (1996).

${ }^{24}$ L. Vriens, Phys. Lett. 8, 260 (1964).

${ }^{25}$ S. Rauf and M. J. Kushner, J. Appl. Phys. 85, 3460 (1999).

${ }^{26}$ M. Ohwa and M. Obara, J. Appl. Phys. 59, 32 (1986).

${ }^{27}$ Y. J. Shui and M. A. Biondi, Phys. Rev. A 17, 868 (1978).

${ }^{28}$ M. A. Biondi, in Principles of Laser Plasmas, edited by G. Bekefi (Wiley Interscience, New York, 1976), Chap. 4.

${ }^{29}$ V. A. Sheverev, V. P. Stepaniuk, and G. G. Lister, J. Appl. Phys. 92, 3454 (2002).

${ }^{30}$ H. W. Ellis, R. Y. Pai, E. W. McDaniel, E. A. Mason, and L. A. Viehland, At. Data Nucl. Data Tables 17, 177 (1976).

${ }^{31}$ D. Uhrlandt and St. Franke, J. Phys. D 35, 680 (2002).

${ }^{32}$ T. Holstein, Phys. Rev. 72, 1212 (1947); 83, 1159 (1951).

${ }^{33}$ J. Dutton, in Electrical Breakdown of Gases, edited by J. M. Meek and J. D. Craggs (Wiley-Interscience, Norwich, 1978), Chap. 3.

${ }^{34}$ N. P. Ostrom (private communication, 2003).

${ }^{35}$ C. Penache, M. Miclea, A. Bräuning-Demian, O. Hohn, S. Schössler, T. Jahnke, K. Niemax, and H. Schmidt-Böcking, Plasma Sources Sci. Technol. 11, 476 (2002). 